Synthèse bibliographique

\title{
LE COLMATAGE MINÉRAL DU LIT DES COURS D'EAU : REVUE BIBLIOGRAPHIQUE DES MÉCANISMES ET DES CONSÉQUENCES SUR LES HABITATS ET LES PEUPLEMENTS DE MACROINVERTÉBRÉS.
}

\author{
S. GAYRAUD (1, 3), E. HÉROUIN (2), M. PHILIPPE (1)
}

(1) Cemagref, Unité de Recherche Biologie des Ecosystèmes Aquatiques, Laboratoire d'Hydroécologie Quantitative, 3 Bis Quai Chauveau, 69336 LYON Cedex 09, France.

(2) Cemagref, Unité de recherche Hydrologie-Hydraulique, 3 Bis Quai Chauveau, 69336 LYON Cedex 09, France.

(3) Adresse actuelle : ESA/CNRS 5023, Laboratoire d'écologie des eaux douces et des grands fleuves, Université Lyon 1, $43 \mathrm{Bd}$ du 11 Novembre 1918, 69622 VILLEURBANNE Cedex, France. E-mail : sebastien.gayraud@univ-lyon1.fr

\section{RÉSUMÉ}

Le développement des activités humaines a conduit dans de nombreuses régions à une augmentation du transfert de sédiments fins vers les cours d'eau, multipliant les phénomènes de colmatage. Le colmatage fait référence au dépôt de sédiments organiques ou minéraux et à leur infiltration dans le benthos et l'hyporhéos. Dans cet article de synthèse, le colmatage par les sédiments minéraux est présenté du point de vue de ses mécanismes et de sa variabilité spatio-temporelle dans un schéma d'organisation global du cours d'eau. Le colmatage entraîne une modification des habitats benthique et interstitiel, ainsi que des échanges d'eau et de matières entre la surface et l'hyporhéos. II affecte la structure du peuplement d'invertébrés en modifiant la structure et la stabilité du substrat, la disponibilité des ressources trophiques et de l'oxygène, ainsi que de la pression de prédation. Les conséquences à court terme sont une augmentation de la dérive et une réduction de l'abondance totale des organismes. Sur le long terme le colmatage affecte la survie, le développement et la croissance des invertébrés ainsi que la biomasse et la productivité du peuplement. Les espèces sensibles adaptées aux substrats grossiers disparaissent au profit des espèces adaptées aux sédiments fins. Les conséquences sur la faune hyporhéique sont peu connues mais l'importance de l'hyporhéos dans le fonctionnement du cours d'eau suggère que son altération pourrait avoir des conséquences importantes en terme de capacité de résilience du peuplement face aux perturbations. (MES).

Mots-clés : substrat, hyporhéos, sédiment, invertébrés, matières en suspension 


\title{
THE CLOGGING OF STREAM BEDS: A REVIEW OF MECHANISMS AND CONSEQUENCES ON HABITATS AND MACROINVERTEBRATE COMMUNITIES.
}

\begin{abstract}
The clogging refers to deposition and infiltration of fine organic and inorganic sediments downward within the hyporheic zone. In this review, we presented clogging mechanisms and their spatio-temporal variability in the context of a global stream functioning. Clogging modifies stream bottom and interstitial habitats characteristics and reduces the exchange of water and dissolved substances between the stream surface and the hyporheic zone. Modifications of substrate structure, substrate stability, oxygen and trophic resources and predator influence lead to changes in community structure. In a first step, drift increases whereas the invertebrate abundance drops. In a second step, likelihood to survive, growing, standing crop and productivity are affected. Coarse substrate adapted assemblages are progressively replaced by invertebrates adapted to fine sediments. The studies about consequences of clogging on the hyporheos are scarce, but the major role of the hyporheos on the dynamic of streams suggests that its alteration would strongly decrease the stream resilience capacity after disturbances.
\end{abstract}

Key-words : substrate, hyporheic zone, fine sediments, invertebrates, suspended sediment (SS).

\section{INTRODUCTION}

Le développement récent des activités humaines dans de nombreuses régions du globe est la cause principale de l'accroissement des transferts de sédiments fins (sables, argiles et limons) vers les cours d'eau (WATERS, 1995). Pour ne citer que les principales, l'érosion des bassins versants liée à la déforestation ou aux pratiques culturales, la mauvaise gestion des berges, le déversement des déchets miniers, l'extraction de granulats du lit mineur ainsi que le fonctionnement et l'entretien des barrages et des plans d'eau sont autant de sources de sédiments fins qui conduisent au colmatage des habitats aquatiques (MEADE, 1988 ; WATERS, 1995). A l'heure actuelle, les problèmes posés par les sédiments fins au niveau international sont si préoccupants que leur gestion constitue un critère à part entière dans l'évaluation des débits minima à appliquer pour satisfaire les exigences de la biocénose aquatique. Pour autant, de nombreuses questions se posent encore sur les mécanismes du colmatage et ses conséquences à long terme sur les cours d'eau (WATERS, 1995).

Le colmatage minéral fait référence aux phénomènes de dépôt et d'infiltration de sédiments fins minéraux au sein du lit, ce qui provoque un remplissage des interstices du substrat et conduit à une altération de ses fonctions. Les conséquences biologiques les plus connues de ce type de colmatage sont la réduction des habitats qui conduisent à la réduction des effectifs piscicoles (BJORNN et al., 1977 ; ALEXANDER et HANSEN, 1986) et la réduction de la survie des œufs des lithophiles (CHAPMAN, 1988). La faune piscicole est également affectée par l'intermédiaire des peuplements d'algues et d'invertébrés qui constituent pour celle-ci des ressources trophiques importantes (SCOTT et CROSSMAN, 1973). Chez les macroinvertébrés, le substrat est indispensable à l'accomplissement de nombreuses fonctions biologiques telles que la reproduction, le développement des œufs et l'alimentation (HYNES, 1970 ; MINSHALL, 1984). II influence leur répartition spatiale au niveau local (CUMMINS et LAUFF, 1969), et la structure (RICHARDS et al., 1993) et la productivité du peuplement (WATERS, 1984) au niveau du cours d'eau. Ces nombreuses interactions avec le substrat rendent les macroinvertébrés particulièrement sensibles au colmatage. 
Si le colmatage a été principalement étudié au niveau de l'habitat benthique, sa dimension verticale ne peut plus être négligée aujourd'hui tant le nombre d'études montrant les liens entre la surface et l'hyporhéos s'est accru depuis 3 décennies (BRUNKE et GONSER, 1997). L'hyporhéos constitue un habitat permanent pour les organismes interstitiels (GIBERT et al., 1977 ; MARMONNIER et DOLE, 1986 ; WARD et al., 1998), mais également un habitat temporaire (WILLIAMS, 1984), et une zone refuge durant les crues (DOLE-OLIVIER et al., 1997) et les étiages sévères (BOULTON et LAKE, 1992) pour de nombreux invertébrés benthiques.

Les très nombreux travaux (plus de 3000 références ont été sélectionnées en 1991 par RYAN, 1991) tant expérimentaux que de terrain sur l'impact des matières en suspension (MES) et des dépôts de sédiments fins sur les invertébrés ont été synthétisés au travers de revues bibliographiques très détaillées (CORDONE et KELLY, 1961 ; CHUTTER, 1969 ; LLOYD et al., 1987 ; RYAN, 1991 ; WATERS, 1995). Cet article a été écrit afin de présenter ces connaissances en nous focalisant sur les mécanismes d'action du colmatage d'une part et en recalant ces mécanismes dans une présentation plus globale des relations entre les invertébrés et le substrat d'autre part. Nous aborderons tout d'abord l'organisation et les propriétés du substrat afin de dégager les paramètres susceptibles d'être affectés par le colmatage, avant de présenter dans une seconde partie les mécanismes de dépôt et d'infiltration des sédiments fins qui conduisent au colmatage. Dans une dernière partie, nous aborderons les conséquences du colmatage sur les organisations structurelles et fonctionnelles des peuplements de macroinvertébrés benthique et hyporhéique.

\section{ORGANISATION ET PROPRIÉTÉS DES SUBSTRATS}

La répartition des particules constituant le lit du cours d'eau est contrôlée par l'écoulement, qui produit ainsi une mosaïque de substrats très diversifiée. On distingue fondamentalement les substrats fins relativement fluides en raison d'une forte teneur en eau, des substrats grossiers plus rigides (Figure 1 ; WARD et al., 1998). Ces substrats ont toutefois une organisation commune : les particules de plus grande taille créent une macrostructure (framework) fortement poreuse que viennent combler les particules les plus fines (matrix). Les interstices résultant de cette architecture constituent l'espace interstitiel qui permet le stockage ou la circulation de l'eau, des substances dissoutes, de la matière organique et des organismes vivants (BRUNKE et GONSER, 1997). Par un phénomène d'adsorption, une partie de l'eau interstitielle est piégée par les particules de sédiment (KEMPER et al., 1964) tandis qu'une autre fraction est libre et peut ainsi participer aux échanges entre la surface et la zone hyporhéique. L'eau piégée par adsorption est d'autant plus importante que la taille des particules est petite, expliquant la structure particulièrement fluide des substrats fins. En raison de cette relative fluidité, les invertébrés peuvent s'y mouvoir aisément par fouissage en modifiant aisément l'arrangement des particules. Au contraire, la structure plus rigide de substrats grossiers est plus difficilement modifiable par les invertébrés et leur utilisation sera conditionnée par la taille et la quantité des interstices (GAYRAUD et PHILIPPE, 2001). Cette dernière peut être mesurée par l'intermédiaire de la porosité qui représente le volume d'eau interstitielle non piégée par les particules dans un volume de substrat donné (MARIDET, 1990).

D'un point de vue théorique, les substrats constitués de particules sphériques et de taille homogène ont une porosité maximale variant de 26 à $48 \%$ selon l'architecture du lit. Dans ce type de substrats, la porosité est indépendante du diamètre des particules (FURNAS, 1931) mais la taille des interstices est directement corrélée au diamètre médian de la distribution granulométrique (WILLIAMS, 1972). Dans le cas de substrats grossiers constitués de particules de taille et de forme variables tel que ceux rencontrés dans les cours d'eau (BRETSCHKO, 1994), la porosité est plus faible $(<30 \%)$ et dépend principalement des proportions de chaque classe granulométrique, du tassement des 
particules (WESTMAN et HUGILL, 1930 ; GAYRAUD et PHILIPPE, sous presse), de leur forme (TICKELL et HIATT, 1938) et, dans une moindre mesure, de leur nature géologique en raison des micro-aspérités recouvrant la surface de certaines roches. Globalement, on observe que la porosité des substrats grossiers est d'autant plus faible que la granulométrie est hétérogène et que la proportion d'argiles, de limons et de sables augmente (MARIDET et PHILIPPE, 1995 ; GAYRAUD et PHILIPPE, sous presse).

\section{Figure 1 \\ Deux modes d'arrangement extrêmes des particules au sein du lit sur lesquels est basée la distinction entre substrats fins et substrats grossiers.}

\section{Figure 1}

Two styles of particle arrangement in streams that lead to the distinction between soft and hard substrates.

Verticalement, les 20 premiers centimètres du substrat se comportent comme une interface (au sens de BRETSCHKO dans VERVIER et al., 1997) entre le lit profond (zones hyporhéique et souterraine) et l'écoulement de surface. Cette zone d'interface est constituée d'une couche d'armure composée généralement de matériaux grossiers et d'une couche de sub-surface constituée de matériaux plus fins et plus diversifiés. La couche d'armure constitue une protection de la couche de sub-surface contre les contraintes générées par l'écoulement, mais contrôle en même temps, par son degré d'ouverture, l'intensité des échanges d'eau et de matière entre la surface et le lit profond.

Pour un lit homogène du point de vue morphologique, l'écoulement de l'eau dans le milieu interstitiel est laminaire et sa vitesse est contrôlée par la perméabilité du substrat, la viscosité de l'eau ainsi que par le gradient de pression longitudinal (loi de Darcy). La vitesse dans le milieu interstitiel varie de quelques $\mathrm{mm} /$ heure à quelques $\mathrm{cm} / \mathrm{seconde}$ (WILLIAMS et HYNES, 1974 ; ANGRADI et HODD, 1998 ; WAGNER, 1999). La perméabilité qui exprime l'aptitude au déplacement de l'eau dans les sédiments dépend principalement de la porosité du substrat, de la taille des interstices et de la connectivité entre ces interstices. La viscosité dépend uniquement de la température et diminue lorsque celle-ci augmente. 
A )

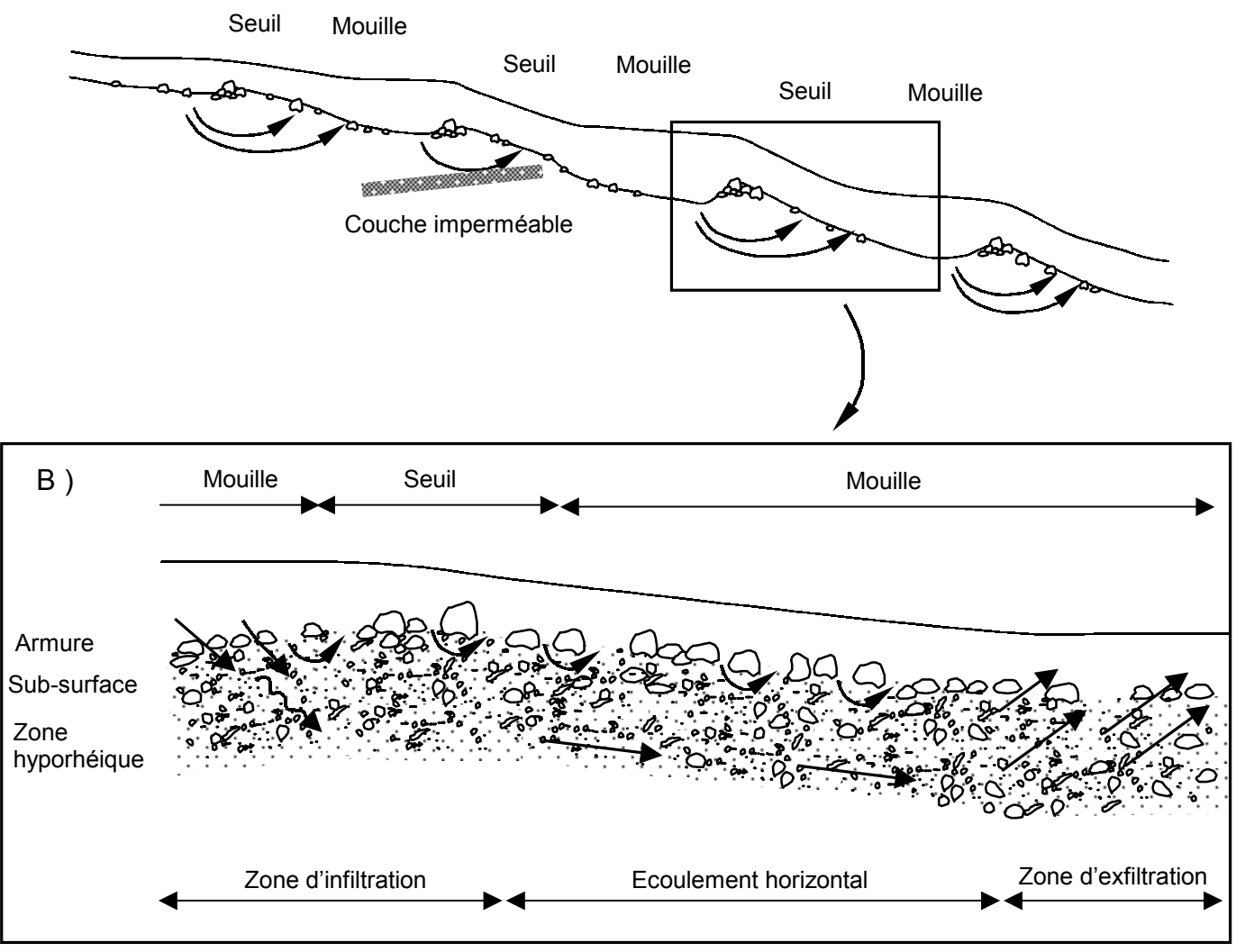

Figure 2

Mouvements d'eau à plusieurs échelles entre la surface et la zone hyporhéique. A) Echanges hydriques induits par les discontinuités géomorphologiques et géologiques. B) Détail des mouvements hydriques au sein d'une séquence mouille-seuil-mouille.

Figure 2

Multi-scale water exchanges between the surface water and the hyporheic zone of streams. A) Water exchange explained by discontinuity of geomorphology and geology. B) Details of water exchanges within a pool-riffle sequence.

Les irrégularités morphologiques que l'on observe dans les cours d'eau, tant à l'échelle locale (hétérogénéité de la perméabilité, présence de grandes particules) qu'à une échelle plus large (faciès d'écoulement), induisent une composante verticale dans l'écoulement de l'eau au sein du milieu interstitiel (Figure 2 ; VAUX, 1968 ; HARVEY et BENCALA, 1993 ; ELLIOT et BROOKS, 1997a, b) qui est prépondérante pour comprendre les mécanismes du colmatage. La présence de particules de grande taille en saillie par rapport au lit induit la pénétration d'eau en amont de ces particules et sa résurgence quelques centimètres en aval (Figure 2 ; THIBODEAUX et BOYLE, 1987 ; VOLLMER et al., 2000). L'alternance de séquences seuil / mouille (MALAVOI, 1989) crée également des mouvements d'eau complexes au sein du lit. L'eau de surface peut pénétrer de manière localisée dans le lit profond en amont d'un seuil (zone d'infiltration), puis ressortir de manière plus diffuse en aval (zone d'exfiltration) après un transit plus ou moins long au sein des sédiments. La correspondance de ces alternances seuil / mouille avec les bancs de galets (DERRUAU, 1974) se traduit par une pénétration de l'eau en tête du banc et une résurgence localisée en queue de banc (MARMONIER et DOLE, 1986 ; DOLE-OLIVIER, 1998). Enfin, les différences de perméabilité des sédiments, par exemple liées à la 
présence de couches géologiques imperméables, peuvent également être à l'origine de mouvements d'eau complexes (VANEK, 1997). Les travaux les plus récents indiquent que les micro-irrégularités du lit induisent de forts échanges dans les 20 premiers centimètres du lit, tandis que la pénétration d'eau dans les couches plus profondes serait induite par les irrégularités du lit à large échelle comme l'alternance des faciès d'écoulement (SAENGER, 2000 ; SAENGER et LENK, 2000).

\section{DÉPÔT ET INFILTRATION DES SÉDIMENTS FINS}

La taille des particules incriminées dans les phénomènes de colmatage apparaît extrêmement variable selon les objectifs des études qui ont été menées (études sur le benthos : $<2 \mathrm{~mm}$; études sur les frayères de Salmonidés : $<0,8 \mathrm{~mm}$; études sur l'hyporhéos : $<0,063 \mathrm{~mm}$ ). Ces particules participent toutes au colmatage mais ces divergences reflètent leur capacité différente à pénétrer le lit des cours d'eau. Au même titre que les sédiments inorganiques, la matière organique particulaire fine participe au colmatage avec toutefois des différences liées à leur faible densité et à leur dégradabilité par la faune (SCHÄLCHLI, 1993 ; BRUNKE, 1999).

\section{Mécanismes de colmatage et de décolmatage}

Le phénomène de colmatage peut être décomposé en plusieurs étapes qui constituent également différents degrés de colmatage du substrat. Les sédiments peuvent simplement se déposer dans les zones où la capacité de transport est réduite (berges, mouilles, annexes du cours d'eau), et/ou bien pénétrer en profondeur dans la zone hyporhéique. Des travaux expérimentaux, permettant de simuler un apport de sédiments fins important et localisé dans le temps ont permis d'identifier les paramètres influençant le dépôt des sédiments, leur profondeur de pénétration dans le lit et le degré de colmatage atteint (BESCHTA et JACKSON, 1979 ; SCHÄLCHLI, 1992, 1993). Le principal paramètre influençant les mécanismes de dépôt et de remise en suspension des fines est la contrainte de cisaillement adimensionnelle $(\theta)$ qui représente le rapport entre les forces responsables de l'entraînement des particules et celles responsables de leur stabilité (MILHOUS et BRADLEY, 1986 ; PETIT, 1989 ; MILHOUS, 1998) :

$$
\theta=\mathrm{RS} /\left[\mathrm{d} 50\left(\mathrm{G}_{\mathrm{s}}-1\right)\right]
$$

où $\mathrm{R}$ est le rayon hydraulique de la section de mesure (approximativement égal à la profondeur moyenne), $\mathrm{S}$ la pente de la ligne d'énergie, d50 est le diamètre médian des matériaux du lit ou de l'armure et $\mathrm{G}_{\mathrm{s}}$ la densité des sédiments en place ( $\mathrm{kg} / \mathrm{litre}$ ). SCHÄLCHLI (1992) montre que la phase de colmatage du lit se produit pour une contrainte $\theta<0,056$. Jusqu'à cette valeur, l'augmentation de la contrainte provoque une obturation des interstices par les sédiments fins et une réduction de la conductivité hydraulique. Bien que cela ne soit pas démontré, SCHÄLCHLI suppose que la pénétration des fines serait causée par un accroissement de la turbulence liée à l'augmentation de la contrainte.

On considère que le décolmatage intervient au delà d'une contrainte adimensionnelle $\theta \cong 0,056$ (SCHÄLCHLI, 1992). Le phénomène se décompose en trois phases successives. 1) Une phase de nettoyage de la couche d'armure se produit lorsque la contrainte atteint une valeur comprise entre 0,056 et 0,072 . Ce nettoyage provoque le départ des particules fines ce qui réduit l'enchâssement des particules les plus grosses qui seront alors mobilisables pour un seuil de contrainte plus bas. 2) La destruction de l'armure intervient lorsque la contrainte atteint la valeur 0,078. En raison du départ des sédiments fins, la conductivité hydraulique du lit atteint alors sa valeur maximale. 3) Le lit est ensuite mobilisé plus ou moins profondément en fonction de la taille des matériaux et de leur compaction, lorsque la contrainte dépasse la valeur 0,078 . 
Dans les cours d'eau, les valeurs critiques de la contrainte adimensionelle peuvent différer des valeurs présentées précédemment à cause de différences d'ordre géomorphologiques ou liées à l'arrangement des particules (KOMAR, 1987 ; PETIT, 1989). Par exemple, la position de saillie de grosses particules par rapport au niveau moyen du lit permet leur départ pour une contrainte plus faible. Au contraire l'imbrication du matériel augmente la contrainte critique pour les particules de petites dimensions. La forme et l'angle de repos des particules ont également une influence, par exemple le dépôt " en tuilage » et la forme aplatie des particules augmentent la contrainte critique nécessaire à la mobilisation de l'ensemble de l'armure. Parce que l'influence de ces facteurs peut être grande et qu'elle est difficilement quantifiable (voir PETIT, 1989), il en résulte une difficulté importante pour calculer avec précision le débit qui permettrait de décolmater le lit (MILHOUS, 1998).

\section{Infiltration dans la zone hyporhéique}

La profondeur de pénétration des particules est influencée par la taille des particules transportées (BESCHTA et JACKSON, 1979), l'homogénéité des particules qui constituent l'armure (BEYER et BANSCHER, 1975 dans BRUNKE, 1999) et la taille des pores (FROSTICK et al., 1984). Les auteurs considèrent que lors d'un apport ponctuel de sédiments fins, la profondeur maximale de pénétration varie entre 2,5 et 5 fois le d90 (diamètre non dépassé par $90 \%$ des particules) de l'armure, en fonction du diamètre des sédiments fins et des conditions d'écoulement. Dans des conditions expérimentales, BESCHTA et JACKSON (1979) puis SCHÄLCHLI (1992) ont montré qu'un apport de sédiments localisé dans le temps n'influence que les 10-15 premiers centimètres car le colmatage de cette zone forme une barrière qui se comporte comme un filtre et empêche la pénétration en profondeur des particules plus fines. Toutefois, compte tenu de la durée et de la répétition des apports de sédiments qui se produisent en milieu naturel, ceux-ci migrent probablement plus profondément, ce qui conduit à leur stockage en profondeur (LEICHTFREID, 1988 ; SEAR, 1993 ; PANEK, 1994 ; BRUNKE, 1999). De plus, en milieu naturel, le phénomène de filtration ne limite pas la profondeur de pénétration des fines mais conduit à un gradient vertical de sédiments fins (JOBSON et CAREY, 1989), les plus grossiers $(0,063-2 \mathrm{~mm})$ étant plus abondants en surface et les plus fins $(<0,003 \mathrm{~mm}$, 0,003-0,063 $\mathrm{mm}$ ) en profondeur.

Le rôle des crues est important dans la dynamique des sédiments fins. Sur un cours d'eau de Nouvelle-Zélande, MATTHAEl et al. (1999) ont montré que les crues survenant 6 fois dans l'année affectaient le lit uniquement sur les 10-15 cm les plus superficiels, suggérant que l'action remaniante des crues est plus forte à la surface du lit qu'en profondeur. Les sédiments constituant l'hyporhéos pourraient n'être remaniés qu'à la faveur d'événements plus rares d'une fréquence proche de celle du débit de plein bord (2 ans ou plus en moyenne), ce qui favoriserait le phénomène de stockage des sédiments fins. De nombreux auteurs (LEOPOLD et al., 1964 ; voir BRAVARD et PETIT, 1997) considèrent d'ailleurs que le débit de plein bord serait optimal pour le façonnement de la géomorphologie du cours d'eau.

Du point de vue spatial, compte tenu des échanges qui se produisent entre la surface et l'hyporhéos, les zones d'infiltration pourraient être plus sensibles au colmatage que les zones d'exfiltration et les zones où l'écoulement est laminaire (JOBSON et CAREY, 1989). En théorie, ces échanges verticaux ont une influence secondaire par rapport aux facteurs cités précédemment (concentration et taille des MES, taille des particules de l'armure...). Néanmoins, en reproduisant en canal expérimental un gradient hydraulique vertical suffisamment fort, SCHÄLCHLI (1992) montre que le colmatage ne se développe pas sur les zones d'exfiltration, contrairement à ce qui se passe dans les zones d'infiltration. De plus, une phase d'exfiltration à la suite d'une phase d'infiltration permet d'expulser les sédiments fins qui se sont accumulés pendant l'infiltration. Afin de tester cette hypothèse, BRUNKE (1999) a mesuré la répartition verticale des sédiments fins dans 
l'hyporhéos d'un cours d'eau suisse. En moyenne, les zones d'exfiltration présentent une concentration en sédiments fins (organiques et inorganiques) maximale à $0,50 \mathrm{~m}$ de profondeur tandis qu'elle est maximale à $1,50 \mathrm{~m}$ dans les zones d'infiltration et les zones à écoulement horizontal. Toutefois la forte variabilité spatiale des observations en milieu naturel ne permet pas d'affirmer de manière certaine le rôle du gradient hydraulique vertical dans la distribution des fines.

\section{Influence de l'activité biologique sur le dépôt de sédiments fins}

La faune aquatique peut influencer localement l'arrangement des sédiments lors d'activités comme la reproduction (KONDOLF et al., 1993), les combats pour occuper l'espace ou les déplacements liés à la recherche de nourriture (STATZNER et al., 1996, 1999). Lors de la reproduction, les Salmonidae nettoient les zones de ponte et expulsent environ $30-35 \%$ des sédiments $<1 \mathrm{~mm}$ lors de la formation de la frayère (KONDOLF et al., 1993). Des organismes de plus petite taille tels que les larves de Dinocras cephalotes (plécoptère) réduisent localement la quantité de sédiments fins comblant les interstices de $30 \%$ (ZANETELL et PECKARSKY, 1996) à $75 \%$ (STATZNER et al., 1996) lors de la recherche de proies. DANIELOPOL (1984) suggère que la digestion de la matière organique fine ou du biofilm bactérien par les invertébrés pourrait réduire les effets du colmatage dans la zone hyporhéique et maintenir élevée la conductivité hydraulique du milieu. Malgré l'absence d'études spécifiques aux milieux aquatiques (mais voir pour le milieu terrestre VAN VLIET et al., 1993), il est aisé de concevoir le rôle dans le maintien de l'espace interstitiel que peuvent avoir les organismes fouisseurs tels que les oligochètes de la famille des Lumbricidae et des Lumbriculidae qui se déplacent en créant des galeries dans le lit. Loin d'être négligeable, cette activité d'ingestion a été évaluée à $12,6 \mathrm{~kg}$ de sédiments fins par gramme de biomasse et par an, chez les oligochètes de la famille des Tubificidae (BREKHOVSKIKH et VISHNEVSKAYA, 1994).

\section{RÉPONSE DU PEUPLEMENT DE MACROINVERTÉBRÉS}

Les apports de sédiments fins influencent le peuplement de macroinvertébrés, soit de manière directe en exerçant un stress (réduction de la luminosité, action mécanique, éléments polluants éventuels) sur les organismes, soit par l'intermédiaire de la disponibilité des ressources trophiques, de l'oxygène et de l'habitat (Figure 3). La réduction de la luminosité consécutive au transport par suspension (KIRK, 1985) peut diminuer la production primaire (GRAHAM, 1989). En se mélangeant au périphyton (GRAHAM, 1990) et à la matière organique particulaire (SLOANE-RICHEY et al., 1981), les sédiments fins réduisent l'attractivité et la qualité énergétique de ces ressources trophiques (RYDER, 1989). La modification et l'homogénéisation de l'habitat engendrée par le colmatage, i.e. la réduction des échanges hydriques, du volume interstitiel et de la taille des interstices, est également prépondérante dans les changements qui interviennent sur le peuplement. Ces changements sont graduels en fonction du degré de colmatage atteint localement, de son étendue spatiale et temporelle, ainsi que des caractéristiques hydrologiques du cours d'eau. Ces dernières contrôlent la capacité de transport solide du cours d'eau et donc sa capacité de retour vers la structure et la dynamique sédimentaire précédant l'apport de sédiments fins.

\section{Conséquences du transport de fines et du colmatage sur le benthos}

\section{Modifications comportementales}

L'augmentation du transport de sédiments par suspension et charriage intervenant soit lors d'une crue, soit lors d'un apport ponctuel artificiel (vidange, extraction de granulats) provoque généralement une augmentation de la mobilité des invertébrés par dérive (GAMMON, 1970 ; WHITE et GAMMON, 1977 ; O'HOP et WALLACE, 1983 ; CULP 
et al., 1986 ; DOEG et MILLEDGE, 1991). La dérive peut être soit la conséquence de l'action mécanique directe des particules (LAPERRIERE et al., 1983) ou de la turbulence sur les organismes (BORCHARDT et STATZNER, 1990), soit un comportement en réponse à la réduction de la luminosité ou à l'apparition de conditions défavorables (RYDER, 1989). D'un point de vue quantitatif, ROSENBERG et WIENS (1978) ont montré expérimentalement qu'une exposition à une concentration de MES de $8 \mathrm{mg} / \mathrm{l}$ (ce qui constitue une augmentation limitée) pendant 2-3 heures induisait une augmentation immédiate de l'intensité de la dérive d'un facteur 3. La réponse des invertébrés n'est pas toujours si nette et pourrait également dépendre de leur rythme d'activité (CULP et al., 1986), ce qui expliquerait l'absence de dérive diurne observée dans certaines études (e.g. CIBOROWSKI et al., 1977).

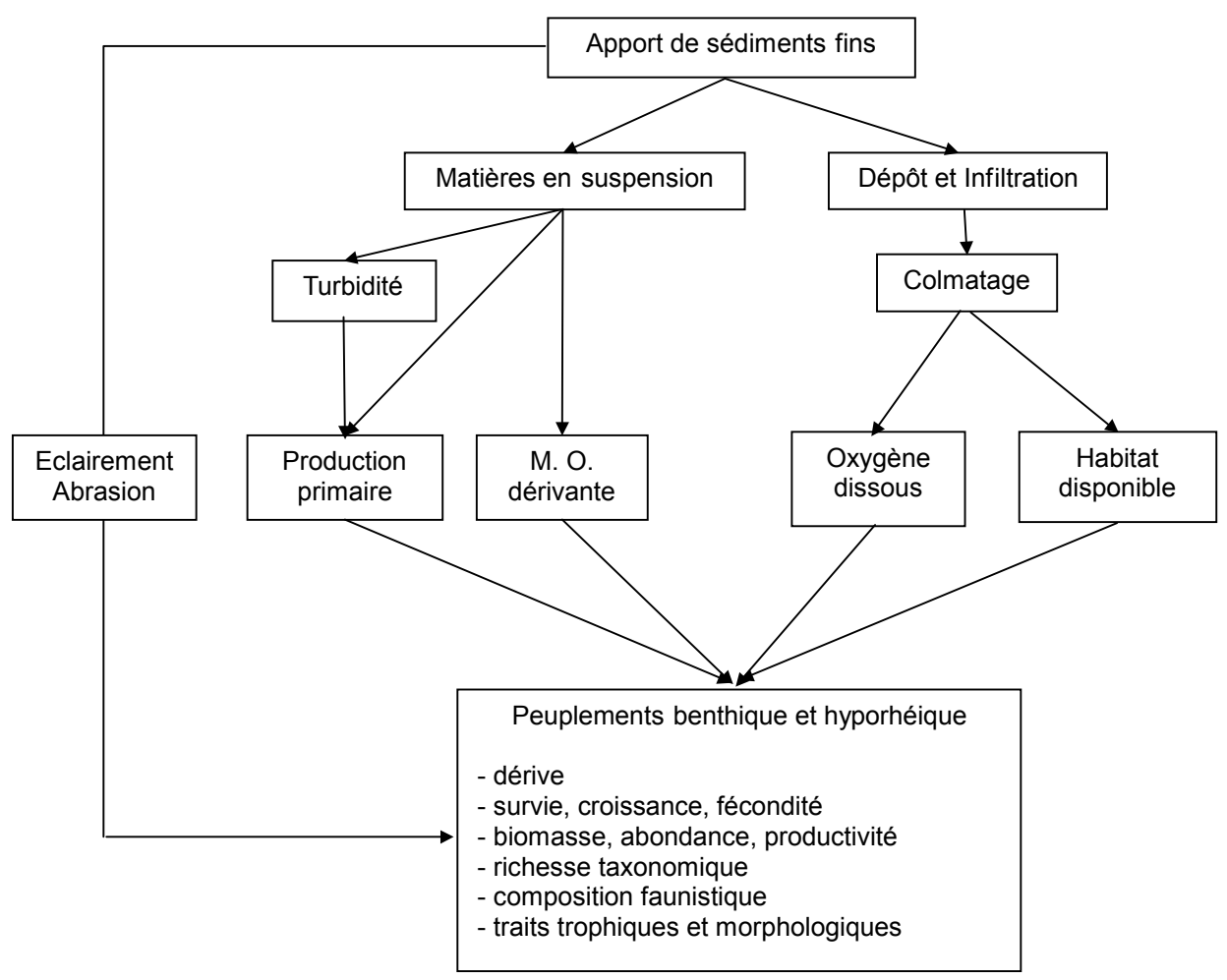

Figure 3

Schéma récapitulant l'influence des apports de sédiments fins sur le peuplement d'invertébrés et sur son habitat.

\section{Figure 3}

Influence of fine sediment increase on habitats and invertebrate communities.

On observe des différences de comportement parmi les invertébrés (principalement les éphéméroptères, plécoptères, trichoptères et coléoptères) qui ont fait l'objet d'expérience. Ainsi, certains plécoptères (e.g. Peltoperles maria dans O'HOP et WALLACE, 1983 ) et de nombreux éphéméroptères (e.g. Seratella subvaria dans CIBOROWSKI et al., 1977 ; Baetis sp., Austrophlebioides sp. dans DOEG et MILLEDGE, 1991 ; Paraleptophlebia sp. dans CULP et al., 1986 et ANGRADI, 1999) adoptent un comportement de dérive évident face à l'augmentation de la concentration en sédiments fins. Au contraire les coléoptères tels Oulimnius sp. (LENAT, 1984), Stenelmis sp. (WHITE et GAMMON, 1977) et des membres de la famille des Helodidae (coléoptères), semblent relativement indifférents à court terme aux fluctuations de la concentration en sédiments fins. 
L'accroissement de la mobilité des invertébrés peut se traduire par une sensibilité accrue à la prédation. Par exemple, BRUSVEN et ROSE (1981) montrent que la prédation de Seratella grandis, Heptagenia solitaria, Rhyacophila vaccua et Hesperoperla pacifica, par le chabot (Cottus rhotheus) est significativement plus forte dans les substrats sableux et les substrats grossiers fortement ensablés.

\section{Réduction de la croissance, de la fécondité et de la survie}

Bien qu'aucune étude ne permette de quantifier la mortalité provoquée par l'action mécanique (abrasion) des sédiments fins, les auteurs considèrent généralement que celleci est faible et que les changements d'abondance résultent de la dérive. Par contre, la réduction de la qualité nutritionnelle des ressources trophiques (périphyton, matière organique particulaire) pourrait réduire la croissance des invertébrés ainsi que leur fécondité et leur survie (GRAHAM, 1989). Ainsi, RYDER (1989) montre que les larves de Pycnocentrodes sp. broutent préférentiellement le périphyton exempt de limon et que la croissance des jeunes stades de ce trichoptère est significativement réduite chez les individus nourris avec du périphyton mélangé avec des limons. La diminution de la concentration en oxygène dissous consécutive à la réduction des échanges entre la surface et le milieu interstitiel peut également influencer le développement et la survie de certains stades de développement (e.g. nymphes d'Hydropsyche, RUTHEFORD et MACKAY, 1986). Par ailleurs celle-ci affecte différemment les taxons et HARRISON et FARINA (1965) montrent qu'en présence de la même concentration de sédiments fins, les œufs de Bulinus globulus ne parviennent pas à maturité tandis que les œufs de Lymnea natalensis se développent normalement.

\section{Modifications faunistiques du peuplement}

Lorsque le recouvrement surfacique des éléments grossiers de l'armure par les fines varie entre $1 / 3$ et $2 / 3$, la réduction de l'habitat interstitiel disponible pour les invertébrés (LENAT et al., 1981 ; WATERS, 1995) conduit généralement à la diminution de l'abondance des éphéméroptères, des plécoptères et des trichoptères (BJORNN et al., 1977 ; LENAT et al., 1979 ; LENAT et al., 1981 ; RICHARDS et al., 1993 ; ANGRADI, 1999), qui constituent la faune caractéristique des substrats grossiers (HYNES, 1970). Parallèlement, on observe une augmentation de l'abondance et de la biomasse de taxons à tendance fouisseuse tels que les oligochètes, les chironomidae et certains mollusques. Lorsque le recouvrement dépasse les $2 / 3$ de la surface des éléments grossiers, LENAT et al. (1981) interprètent cette modification non plus comme une réduction de l'habitat mais comme un changement d'habitat, dans la mesure où la surface favorable à la faune pétricole n'est plus suffisante pour permettre son maintien. Les changements faunistiques évoqués précédemment sont alors amplifiés.

Ces changements faunistiques s'expliquent par des différences de préférence d'habitat entre espèces qui ont été testées expérimentalement (BRUSVEN et PRATHER, 1974 ; MCCLELLAND et BRUSVEN, 1980). Parmi les espèces d'EPT (Ephéméroptères, Plécoptères, Trichoptères) testées, les plécoptères et les trichoptères présentent une préférence très marquée vis-à-vis des substrats non ensablés lorsqu'ils sont soumis à un choix parmi des substrats caractérisés par des niveaux croissants d'ensablement $(1 / 3,1 / 2$, $2 / 3)$, tandis que les éphéméroptères semblent moins sensibles à l'ensablement (notamment Seratella grandis, Rhitrogena robusta, Baetis sp. et Caenis sp.).

Ces préférences d'habitat sont basées sur des comportements et des morphologies résultant probablement d'une évolution adaptative (HYNES, 1970). Par exemple, GAYRAUD et PHILIPPE (2001) montrent que les cours d'eau caractérisés par une faible disponibilité en habitat interstitiel, présentent une plus forte proportion d'invertébrés adaptés morphologiquement à pénétrer le substrat (petite taille, morphologie vermiforme) que les cours d'eau ayant une forte disponibilité en habitat interstitiel. De telles adaptations 
peuvent également être basées sur d'autres exigences écologiques telle que la recherche de ressources trophiques (HYNES, 1970), ou sur des exigences physiologiques telle que le besoin en oxygène (ERIKSEN, 1964).

Conséquences sur l'abondance, la biomasse, la productivité et la diversité du peuplement

A l'échelle de la station, le colmatage se traduit à court terme par une réduction de $10 \%$ (e.g. CLAVEL et al., 1977) à $95 \%$ (e.g. WAGENER et LAPERRIERE, 1985) de l'abondance initiale (NEWCOMBE et MCDONALD, 1991). Sur le long terme, l'évolution dépend de l'hydrologie. Si les phénomènes d'érosion consécutifs à la re-mobilisation des sédiments fins sont rares, un peuplement stable, abondant et composé d'organismes adaptés aux sédiments fins se mettra en place. Au contraire, une fréquence élevée de mobilisation entraînera d'importantes perturbations de l'habitat qui se traduiront par une abondance très fluctuante des effectifs du même peuplement (LENAT et al., 1981). En terme de biomasse, l'influence du colmatage est plus homogène et se traduit par une réduction plus ou moins marquée selon l'étendue spatiale et temporelle du colmatage (WATERS, 1984 ; WATERS, 1995).

La productivité de certaines espèces peut être réduite en réponse à la réduction de la qualité ou de la quantité des ressources trophiques ou bien en réponse à l'incapacité des invertébrés à utiliser ces ressources. Par exemple la réduction de la taille des interstices du substrat affecte l'abondance d'Hydropsyche sp. (GAMMON, 1970) et de Polycentropus kingi (NUTTALL, 1972) qui captent tous deux la matière organique dérivante à l'aide d'un filet de soie. MACKAY et WATERS (1986) ont trouvé une forte différence de production $\left(34,9 \mathrm{~g} \cdot \mathrm{m}^{2}\right.$.an versus $5,8 \mathrm{~g} \cdot \mathrm{m}^{2}$.an) d'Hydropsyche sp. entre deux stations se distinguant par un degré de colmatage différent (la station en amont d'un barrage étant plus ensablée que la station en aval). RYAN (1991) suggère que l'évolution de la productivité globale du peuplement dépend de l'abondance des ressources énergétiques associées aux sédiments fins. Elle sera accrue si l'augmentation des sédiments fins est accompagnée d'une augmentation de la matière organique particulaire comme cela se produit dans les cas d'érosion des terrains agricoles (LENAT, 1984) ou de déforestation.

Le changement d'habitat résultant d'un apport important de sédiments fins se traduit, à l'échelle de la station, par une réduction de la diversité de la mosaïque constituée par les micro-habitats. La disparition des espèces sensibles adaptées aux substrats grossiers consécutive à ce changement n'est pas compensée par l'apparition de nouvelles espèces adaptées aux sédiments fins (qui étaient déjà présentes en petite proportion) ce qui se traduit globalement par une réduction de la richesse taxonomique (WATERS, 1995 ; e.g. NUTTALL, 1972).

\section{Influence de l'infiltration des sédiments fins sur l'hyporhéos}

II existe à l'heure actuelle peu d'études (e.g. BOU, 1977 ; RICHARDS et BACON, 1994 ; BOULTON et al., 1997) permettant de cerner les effets du colmatage de la zone hyporhéique sur sa faune. La zone hyporhéique constitue pourtant un compartiment essentiel dans le fonctionnement des systèmes aquatiques en raison de la diversité et de l'abondance de sa faune ainsi que de son rôle dans la dynamique du système (BRUNKE et GONSER, 1997 ; WARD et al., 1998). De nombreux invertébrés benthiques (Seratella sp., Caenis sp., Leuctra major, Esolus parallelepipedus, Ceratopogoninae, Chironomidae, Hydracariens) peuvent y être rencontrés jusqu'à plus de $30 \mathrm{~cm}$ de profondeur. De nombreux auteurs (e.g. WILLIAMS et HYNES, 1974 ; POOLE et STEWART, 1976 ; GODBOUT et HYNES, 1982 ; WILLIAMS, 1984) estiment que seulement $20-40 \%$ du benthos (en terme d'abondance) est situé dans les 5 premiers centimètres du lit. 
L'impact sur la faune hyporhéique se produit également par le biais de la réduction de l'habitat et des ressources trophiques, ainsi que par la réduction du degré d'oxygénation. Plusieurs auteurs (MARIDET et al., 1992 ; RICHARDS et BACON, 1994 ; MARIDET et al., 1996) ont constaté à l'échelle du micro-habitat, la réduction de l'abondance et du nombre de taxons en relation avec la réduction de la porosité ou l'augmentation de la proportion de sédiments fins. Parmi la faune hyporhéique, ce sont principalement les organismes benthiques qui sont affectés par le colmatage tandis que de nombreux taxons strictement hypogés semblent se satisfaire de concentrations en oxygène plus réduites (BOULTON et al., 1997 ; MALARD et HERVANT, 1999). Compte tenu de l'importance de la zone hyporhéique dans le fonctionnement du système (e.g. potentiel de recolonisation), son colmatage pourrait donc avoir des conséquences importantes sur la dynamique des invertébrés ainsi que sur les niveaux trophiques supérieurs.

\section{CONCLUSIONS ET PERSPECTIVES}

Si les mécanismes de colmatage sont bien compris en situation expérimentale, il existe à l'heure actuelle peu de mesures de terrain permettant d'évaluer en quoi ces mécanismes diffèrent dans les systèmes naturels qui sont souvent bien plus complexes. De nombreuses questions se posent sur la dynamique spatiale et temporelle des sédiments fins en relation avec la géomorphologie et l'hydrologie, ainsi que sur les problèmes d'évolution du système et de réversibilité des effets lorsque les apports de sédiments fins sont accrus de manière importante. L'influence des sédiments fins sur l'hyporhéos a été peu étudiée, de sorte que l'on connaît mal leurs conséquences à long terme sur cet habitat. Au contraire, l'impact des sédiments fins sur le benthos a été évalué à de nombreuses reprises, en différentes situations (expérimentales et études de terrain) et pour différents scénarii (pollution par les mines, la déforestation, les carrières, l'extraction de matériaux, les vidanges de barrage). Ces études ont permis une compréhension globale des mécanismes sur lesquels agissent l'apport de sédiment fins. Toutefois, compte tenu de la complexité de la réponse du peuplement d'invertébrés et de la diversité des situations étudiées, il reste difficile d'identifier les mécanismes prépondérants qui conduisent aux changements faunistiques que nous avons présentés. Une piste intéressante qui permettrait d'intégrer cette complexité réside dans l'utilisation de traits biologiques décrivant les caractéristiques morphologiques (e.g. taille maximale, forme du corps), physiologiques (e.g. durée de vie, modalités de reproduction, organes de résistance) et comportementales (e.g. relations au substrat, préférences trophiques) des invertébrés (STATZNER et al., 1994 ; DOLEDEC et al., 1999 ; STAZNER et al., 2001). Les traits du peuplement qui seront modifiés dans les situations de colmatage permettront d'identifier les principaux mécanismes responsables des changements faunistiques.

\section{REMERCIEMENTS}

Les auteurs remercient Marc Babut, Hervé Capra, Franck Cattanéo, Marie-José Dole-Olivier, Nicolas Lamouroux et Yves Souchon pour les commentaires apportés à une version précédente du manuscrit, ainsi que les trois lecteurs externes désignés par la revue. Cette étude a bénéficié du soutien financier du Ministère de la Recherche, de l'Agence de l'Eau Rhône-Méditérannée-Corse, d'Electricité de France et de la Région Rhône-Alpes. 


\section{BIBLIOGRAPHIE}

ALEXANDER G.R., HANSEN E.A., 1986. Sand bed load in a Brook Trout stream. N. Am. J. Fish. Manage., 6, 9-23.

ANGRADI T.R., 1999. Fine sediment and macroinvertebrate assemblages in Appalachian streams: a field experiment with biomonitoring applications. J. N. Am. Benthol. Soc., $18,49-66$.

ANGRADI T.R., HOOD R., 1998. An application of the plaster dissolution method for quantifying water velocity in the shallow hyporheic zone of an Appalachian stream system. Freshwat. Biol., 39, 301-315.

BESCHTA R.L., JACKSON W., 1979. The intrusion of fine sediments into a stable gravel bed. J. Fish. Res. Board Can., 36, 204-210.

BEYER W., BANSHER E., 1975. Zur kolmation der gewässerbetten bei der uferfiltratgewinnung. Z. Angewandte Geologie, 12, 565-570.

BJORNN T.C., BRUSVEN M.A., MOLNAU M.P., MILLIGAN J.H., KLANT J.H., CHACHO E., SCHAYE C., 1977. Transport of granitic sediment in streams and its effects on insects and fish. Rapport B-036-IDA, College of forestry, wildlife and range sciences, $43 \mathrm{p}$.

BORCHARDT D., STATZNER B., 1990. Ecological impact of urban storm water runoff studied in experimental flumes: population loss by drift and availability of refugial space. Aquat. Sci., 52, 299-314.

BOU C., 1977. Conséquences écologiques de l'extraction des alluvions récentes dans le cours moyen du Tarn. Bull. Ecol., 8, 435-444.

BOULTON A.J., LAKE P.S., 1992. The ecology of two intermittent stream in Victoria, Australia: temporal changes in faunal composition. Freshwat. Biol., 27, 123-138.

BOULTON A.J., SCARSBROOK M.R., QUINN J.M., BURREL G.P., 1997. Land-use effects on the hyporheic ecology of five streams near Hamilton, New Zealand. N. Zeal. J. Mar. Fresh., 31, 609-622.

BRAVARD J.P., PETIT F., 1997. Les cours d'eau : dynamique du système fluvial. Armand Colin/Masson, Paris, $221 \mathrm{p}$.

BREKHOVSKIKH V.F., VISHNEVSKAYA G.N., 1994. The effect of macrobenthos on the mass exchange at the water-sediment interface (review). Water Resour., 21, 301-307.

BRETSCHKO G., 1994. Bedsediment extension, grain shape and size distribution. Verh. Internat. Verein. Limnol., 25, 1631-1635.

BRUNKE M., 1999. Colmation and depth filtration within streambeds: retention of particles in hyporheic interstices. Int. Rev. Hydrobiol., 84, 99-117.

BRUNKE M., GONSER T., 1997. The ecological significance of exchange processes between rivers and groundwater. Freshwat. Biol., 37, 1-33.

BRUSVEN M.A., PRATHER K.V., 1974. Influence of stream sediments on distribution of macrobenthos. J. Entomolol. Soc. Brit. Columbia, 71, 25-32.

BRUSVEN M.A., ROSE S.T., 1981. Influence of substrate composition and suspended sediment on insect predation by the torrent sculpin, Cottus rhoteus. Can. J. Fish. Aquat. Sci., 38, 1444-1448.

CHAPMAN D.W., 1988. Critical review of variables used to define effects of fines in redds of large salmonids. Trans. Am. Fish. Soc., 117, 1-21.

CHUTTER F.M., 1969. The effects of silt and sand on the invertebrate fauna of streams and rivers. Hydrobiologia, 34, 57-76.

CIBOROWSKI J.J.H., POINTING P.J., CORKUM L.D., 1977. The effects of current velocity and sediment on the drift of the mayfly Ephemerella subvaria Mcdunnough. Freshwat. Biol., 7, 567-572.

CLAVEL P., CUINAT R., HAMON Y., ROMANEIX C., 1977. Effets des extractions de matériaux alluvionnaires sur l'environnement aquatique dans les cours supérieurs de la Loire et de l'Allier. Bull. Fr. Pêche Piscic., 268, 121-154.

CORDONE A.J., KELLEY D.W., 1961. The influence of inorganic sediment on the aquatic life of streams. California Fish and Game, 189-228. 
CULP J.M., WRONA F.J., DAVIES R.W., 1986. Response of stream benthos and drift to fine sediment deposition versus transport. Can. J. Fish. Aquat. Sci., 64, 1345-1351.

CUMMINS K.W., LAUFF G.H., 1969. The influence of substrate particle size on the microdistribution of stream macrobenthos. Hydrobiologia, 34, 145-181.

DANIELOPOL D.L., 1984. Ecological investigations on the alluvial sediments of the Danube in the Vienna area phreatobiological project. Verh. Internat. Verein. Limnol., 22, 1755-1761.

DERRUAU M., 1974. Précis de géomorphologie. Masson éditeurs, Paris, 453 p.

DOEG T.J., MILLEDGE G.A., 1991. Effect of experimentally increasing concentrations of suspended sediment on macroinvertebrate drift. Aust. J. Mar. Freshwat. Res., 42, 519-526.

DOLEDEC S., STATZNER B., BOURNAUD M., 1999. Species traits for future biomonitoring across ecoregions: patterns along a human-impacted river. Freshwat. Biol., 42, 737-758.

DOLE-OLIVIER M.J., 1998. Surface water-groundwater exchanges in three dimensions on a backwater of the Rhône River. Freshwat. Biol., 40, 93-109.

DOLE-OLIVIER M.J., MARMONIER P., BEFFY J.L., 1997. Response of invertebrates to lotic disturbance: is the hyporheic zone a patchy refugium. Freshwat. Biol., 37, 257-276.

ELLIOTT A.H., BROOKS N.H., 1997a. Transfer of nonsorbing solutes to a streambed with bed forms: theory. Water Resour. Res., 33, 123-136.

ELLIOTT A.H., BROOKS N.H., 1997b. Transfer of nonsorbing solutes to a streambed with bed forms: laboratory experiments. Water Resour. Res., 33, 137-151.

ERIKSEN C.H., 1964. The influence of respiration and substrate upon the distribution of burrowing mayfly naiads. Verh. Internat. Verein. Limnol., 15, 903-911.

FROSTICK L.E., LUCAS P.M., REID I., 1984. The infiltration of fine matrices into coarsegrained alluvial sediments and its implications for stratigraphical interpretation. J. Geol. Soc., 41, 955-965.

FURNAS C.C., 1931. Grading Aggregates I-Mathematical relations for beds of broken solids of maximum density. Ind. Eng. Chem., 23, 1052-1058.

GAMMON J.R., 1970. The effects of inorganic sediment on stream biota. Rapport 1805DWC12/70, Université de Depauw (USA), $142 \mathrm{p}$.

GAYRAUD S., PHILIPPE M., 2001. Does subsurface interstitial space influence general features and morphological traits of the benthic macroinvertebrate community in streams? Arch. Hydrobiol., 151, 667-686.

GAYRAUD S., PHILIPPE M., sous presse. Influence of bed-sediment features on the interstitial habitat available for stream macroinvertebrates in 15 French streams. Int. Rev. Hydrobiol.

GIBERT J., GINET R., MATHIEU J., REYGROBELLET. J.L., SEYED-REIHANI A., 1977. Structure et fonctionnement des écosystèmes du Haut-Rhône français : IV : Le peuplement des eaux phréatiques ; premiers résultats. Annls. Limnol., 13, 83-97.

GODBOUT L., HYNES H.B.N., 1982. The three dimensional distribution of the fauna in a single riffle in a stream in Ontario. Hydrobiologia, 97, 87-96.

GRAHAM A.A., 1989. The effects of siltation and flow characteristics on the productivity and composition of lotic periphyton. Thèse, Université d'Otago, $253 \mathrm{p}$.

GRAHAM A.A., 1990. Siltation of stone surface periphyton in rivers by clay-sized particles from low concentrations in suspension. Hydrobiologia, 199, 107-115.

HARRISON A.D., FARINA T.D.W., 1965. A naturally turbid water with deleterious effects on the egg capsules of planorbid snails. Ann. Trop. Med. Parasit., 59, 327-330.

HARVEY J.W., BENCALA K.E., 1993. The effect of streambed topography on surfacesubsurface water exchange in mountain catchments. Water Resour. Res., 29, 89-98.

HYNES H.B.N., 1970. The ecology of running waters. Liverpool University Press, Liverpool. $555 \mathrm{p}$.

JOBSON H.E., CAREY W.P., 1989. Interaction of fine sediments with alluvial streambeds. Water Resour. Res., 25, 135-140. 
KEMPER W.D., MAASLAND D.E., PORTER L.K., 1964. Mobility of water adjacent to mineral surfaces. Soil Sci. Soc. Am. Proc., 28, 164-167.

KIRK J.T.O., 1985. Effects of suspendoids (turbudity) on penetration of solar radiation in aquatic ecosystems. Hydrobiologia, 125, 195-208.

KOMAR P.D., 1987. Selective entrainment by a current from a bed of mixed sizes: a reanalysis. J. Sedim. Petrol., 57, 203-211.

KONDOLF G.M., SALE M.J., WOLMAN M.G., 1993. Modification of fluvial gravel size by spawning salmonids. Water Resour. Res., 29, 2265-2274.

LAPERRIERE J.D., BJERKIE D.M., SIMMONS R.C., VAN NIEUWENHUYSE E., WAGENER S.M., REYNOLDS J.B., 1983. Effects of gold mining on interior Alaska stream ecosystems. Rapport IWR-105, Université de l'Alaska, 52 p.

LEICHTFRIED M., 1988. Bacterial substrates in gravel beds of a second order alpine stream (Project Ritrodat-Lunz, Austria). Verh. Internat. Verein. Limnol., 23, 1325-1332.

LENAT D.R., 1984. Agriculture and stream water quality: a biological evaluation of erosion control practices. Environ. Manage., 8, 333-344.

LENAT D.R., PENROSE, D.L., EAGLESON K.W., 1979. Biological evaluation of non-point source pollutants in North Carolina streams and rivers. North Carolina Department of Natural Resources and Community Development, Biological Series 102, Raleigh.

LENAT D.R., PENROSE D.L., EAGLESON K.W., 1981. Variable effects of sediment addition on stream benthos. Hydrobiologia, 79, 187-194.

LEOPOLD L.B., WOLMAN, M.G., MILLER J.P., 1964. Fluvial processes in geomorphology. Freeman, San Fransisco, $522 \mathrm{p}$.

LLOYD D.S., KOENINGS J.P., LAPERRIERE J.D., 1987. Effects of turbidity in fresh waters of Alaska. N. Am. J. Fish. Manage., 7, 34-45.

MACKAY R.J., WATERS T.F., 1986. Effects of small impoundments on Hydropsychid caddis fly production in Valley Creek, Minnesota. Ecology, 67, 1680-1686.

MALARD F., HERVANT F., 1999. Oxygen supply and the adaptations of animals in groundwater. Freshwat. Biol., 41, 1-30.

MALAVOI J.R., 1989. Méthodologie de description et quantification des variables morphodynamiques d'un cours d'eau a fond caillouteux. Rev. Géo. Lyon, 64, 252-259.

MARIDET L., 1990. Relations quantitatives entre les invertébrés, la structure du substrat et les facteurs trophiques. Rapport de DEA, Université Claude Bernard, Lyon I (France), $35 \mathrm{p}$.

MARIDET L., WASSON J.G., PHILIPPE M., 1992. Vertical distribution of fauna in the bed sediment of three running water sites: influence of physical and trophic factors. Regul. Rivers: Res. Manage., 7, 45-55.

MARIDET L., PHILIPPE M., 1995. Influence of substrate characteristics on the vertical distribution of stream macroinvertebrates in the hyporheic zone. Folia. Fac. Sci. Nat. Univ. Masarykianae Brunensis, Biologia, 21, 101-105.

MARIDET L., PHILIPPE M., WASSON J.G., MATHIEU J., 1996. Spatial and temporal distribution of macroinvertebrates and trophic variables within the bed sediment of three streams differing by their morphology and riparian vegetation. Arch. Hydrobiol., 136, 41-64.

MARMONIER P., DOLE M.J., 1986. Les amphipodes des sédiments d'un bras court-circuité du Rhône. Sciences de l'eau, 5, 461-486.

MATTHAEI C.D., PEACOCK K.A., TOWNSEND C.R., 1999. Scour and fill patterns in a New Zealand stream and potential implications for invertebrate refugia. Freshwat. Biol., 42, 41-57.

MCCLELLAND W.T., BRUSVEN M.A., 1980. Effects of sedimentation on the behavior and distribution of riffle insects in a laboratory stream. Aquat. Insects, 2, 161-169.

MEADE R.H., 1988. Movement and storage of sediment in river systems. In : Physical and Chemical Weathering in Geochemical cycles, 165-179.

MILHOUS R.T., 1998. Modelling of instream flow needs: the link between sediment and aquatic habitat. Regul. Rivers: Res. Manage., 14, 79-94. 
MILHOUS R.T., BRADLEY J.B., 1986. Physical habitat simulation and the moveable bed. In : Water Forum'86: World Water Issues in Evolution, 1976-1983. KLINGEMAN P.C., BESCHTA R., KOMAR P. et BRADLEY J.B. (Eds), New York, 1976-1983.

MINSHALL G.W., 1984. Aquatic insect-substratum relationships. In : Ecology of aquatic insects. RESH V.M., ROSENBERG D.M. (Eds.), 358-400.

NEWCOMBE C.P., MACDONALD D.D., 1991. Effects of suspended sediments on aquatic ecosystems. N. Am. J. Fish. Manage, 11, 72-82.

NUTTALL P.M., 1972. The effects of sand deposition upon the macroinvertebrate fauna of the River Camel, Cornwall. Freshwat. Biol., 2, 181-186.

O'HOP J., WALLACE J.B., 1983. Invertebrate drift, discharge, and sediment relations in a southern Appalachian headwater stream. Hydrobiologia, 98, 71-84.

PANEK K.L., 1994. Depth boundaries of bed sediments in an Alpine stream (Project Ritrodat-Lunz, Austria). Verh. Internat. Verein. Limnol., 25, 1646-1648.

PETIT F., 1989. Evaluation des critères de mise en mouvement et de transport de la charge de fond en milieu naturel. Bull. Soc. Géo. Liège, 25, 91-111.

POOLE W.C., STEWART K.W., 1976. The vertical distribution of macrobenthos within the substratum of the Brazos River, Texas. Hydrobiologia, 50, 151-160.

RICHARDS C., BACON K.L., 1994. Influence of fine sediment on macroinvertebrate colonization of surface and hyporheic stream substrates. Great Basin Nat., 54, 106-113.

RICHARDS C., HOST G.E., ARTHUR J.W., 1993. Identification of predominant environmental factors structuring stream macroinvertebrate communities within a large catchment. Freshwat. Biol., 29, 285-294.

ROSENBERG D.M., WIENS A.P., 1978. Effects of sediment addition on macrobenthic invertebrates in a northern Canadian river. Water Res., 12, 753-763.

RUTHEFORD J.E., MACKAY R.J., 1986. Patterns of pupal mortality in field populations of Hydropsyche and Cheumatopsyche (Trichoptera: Hydropsychidae). Freshwat. Biol., 16, 337-350.

RYAN P.A., 1991. Environmental effects of sediments on New Zealand streams: a review. N. Zeal. J. Mar. Freshwat. Res., 25, 207-221.

RYDER G.I., 1989. Experimental studies on the effects of fine sediment on lotic invertebrates. Thèse, Université d'Otago, $216 \mathrm{p}$.

SAENGER N., 2000 Identifikation von austauschprozessen zwischen fließgewässer und hyporheischer zone. Rapport pour l'obtention du diplome d'ingénieur, Technische Universität Darmstadt, $110 \mathrm{p}$.

SAENGER N., LENK M., 2000 Exchange processes in the river bed and their influence on temperature variations. Verh. Internat. Verein. Limnol., 27, 427-430.

SCHÄLCHLI U., 1992. The clogging of coarse gravel river beds by fine sediment. Hydrobiologia, 235/236, 189-192.

SCHÄLCHLI U., 1993. Basic equations for siltation of riverbeds. J. Hydraul. Eng., 121, 274-287.

SCOTT W.B., CROSSMAN E.J., 1973. Freshwater fishes of Canada. Fish. Res. Board Can. Bull., 184, 1-966.

SEAR D.A., 1993. Fine sediment infiltration into gravel spawning beds within a regulated river experiencing floods: ecological implications for salmonids. Regul. Rivers: Res. Manage., 3, 267-275.

SLOANE-RICHEY J., PERKINS M.A., MALUEG K.W., 1981. The effects of urbanization and stormwater runoff on the food quality in two salmonid streams. Verh. Internat. Verein. Limnol., 21, 812-818.

STATZNER B., RESH V.H., DOLEDEC S., 1994. Ecology of the Upper Rhône River: a test of habitat templet theories. Freshwat. Biol., 31 (3), numéro spécial.

STATZNER B., FUCHS U., HIGLER L.W.G., 1996. Sand erosion by mobile predaceous stream insects: implications for ecology and hydrology. Water Resour. Res., 32, 2279-2287. 
STATZNER B., ARENS M.F., CHAMPAGNE J.Y., MOREL R., HEROUIN E., 1999. Silkproducing stream insects and gravel erosion: significant biological effects on critical shear stress. Water Resour. Res., 35, 3495-3506.

STATZNER B., BIS, B., DOLEDEC S., USSEGLIO-POLATERA P., 2001 Perspectives for biomonitoring at large scales: a unified measure for the functional composition of invertebrate communities in European running waters. Basic Appl. Ecol., 2, 73-85.

THIBODEAUX L.J., BOYLE J.D., 1987. Bedform-generated convective transport in bottom sediment. Nature, 325, 341-343.

TICKELL F.G., HIATT W.N., 1938. Effect of angularity of grains on porosity and permeability of unconsolidated sands. APG Bulletin, 22, 1272-1274.

VANEK V., 1997. Heterogeneity of groundwater-surface water ecotones. In : Groundwater/surface water ecotones: Biological and hydrological interactions and management options, GIBERT J., MATHIEU J., FOURNIER F. (Eds.), Cambridge University Press, Cambridge, 151-161.

VAN VLIET P.C.J., WEST L.T., HENDRIX P.F., COLEMAN D.C., 1993. The influence of Enchytraeidae (Oligochaeta) on the soil porosity of small microcosms. Geoderma, 56, 287-299.

VAUX W.G., 1968. Intragravel flow and interchange of water in a streambed. Fish. Bull., 66, 479-489.

VERVIER P., VALETT M.H., HAKENKAMP C.C., DOLE-OLIVIER M.J., 1997. Contribution of the groundwater/surface water ecotone concept to our knowledge of river ecosystem functioning. In : Groundwater/surface water ecotones: Biological and hydrological interactions and management options. GIBERT J., MATHIEU J., FOURNIER F. (Eds.), Cambridge University Press, Cambridge, 238-242.

VOLLMER S., TRÄBING K., DITTRICH A., 2000. Turbulence induced penetration of near bottom water into porous medium-experimental study. In : International symposium on River Flood Defence, Kassel, 17-24.

WAGENER S.M., LAPERRIERE J.D., 1985. Effects of place mining on the invertebrate communities of interior Alaska streams. Freshwat. Biol., 4, 208-214.

WAGNER F.H., 1999. Hyporheic flow in a low order mountain stream. Jahresbericht 19951998 Biologische Station Lunz, 16, 29-34.

WARD J.V., BRETSCHKO G., BRUNKE M., DANIELOPOL D., GIBERT J., GONSER T., HILDREW A.G., 1998. The boundaries of river systems: the metazoan perspective. Freshwat. Biol., 40, 531-569.

WATERS T.F., 1984. Annual production by Gammarus pseudolimnaeus among substrate types in Valley Creek, Minnesota. Am. Mid. Nat., 112, 95-102.

WATERS T.F., 1995. Sediment in streams: sources, biological effects and control. Am. Fish. Soc., Betheda, $251 \mathrm{p}$.

WESTMAN A.E.R., HUGILL H.R., 1930. The packing of particles. J. Am. Ceramic Soc., 13, 767-779.

WHITE D.S., GAMMON J.R., 1977. The effect of suspended solids on macroinvertebrate drift in an Indiana Creek. Ecology, 86, 182-188.

WILLIAMS D.D., 1984. The hyporheic zone as an habitat for aquatic insects and associated arthropods. In : The ecology of aquatic insects. RESH V.H., ROSENBERG D.M. (Eds.), Praeger, New York, 430-455.

WILLIAMS D.D., HYNES H.B.N., 1974. The occurrence of benthos deep in the substratum of a stream. Freshwat. Biol., 4, 233-526.

WILLIAMS R., 1972. The abundance and biomass of the interstitial fauna of a graded series of shell-gravels in relation to the available space. J. Anim. Ecol., 41, 623-646.

ZANETELL B.A., PECKARSKY B.L., 1996. Stoneflies as ecological engineers - hungry predators reduce fine sediments in stream beds. Freshwat. Biol., 36, 569-577. 
